From Institut Municipal d'Investigacions Mèdiques-Hospital del Mar, Barcelona; Institut Català d'Oncologia; Hospital Universitari de Bellvitge, L'Hospitalet de Llobregat; Instituto Oncológico de Guipúzcoa, San Sebastián; Hospital Clínico Universitario de Salamanca, Salamanca; Capio Hospital General de Catalunya, Sant Cugat del Vallés; Centro Oncológico de Galicia, A Coruña: Hospital Ramon y Cajal, Madrid; Hospital Regional Carlos Haya Málaga; Hospital Virgen del Rocío Sevilla; Fundación Puigvert; Hospital de la Santa Creu i Sant Pau, Barcelona; CIBER en Epidemiología y Salud Pública (CIBERESP); Universitat Autònoma de Barcelona, Bellaterra, Spain

Submitted August 7, 2009; accepted May 7, 2010; published online ahead of print at www.jco.org on October 4 2010

Supported by Instituto de Salud Carlos III Fondo Europeo de Desarrollo Regional Grants No. ETS-PI08/90090 and FIS PI020668, Departament d'Universitats, Recerca i Societat de la Informació-Generalitat de Catalunya Grants No. 2005-SGR-00491 and 2009 SGR 1095, and Agència d'Avaluació de Tecnolgia i Recerca Mèdiques Grant No. $086 / 24 / 2000$. A.P. is supported by a support contract through the Nationa Health System, Instituto Nacional Carlos III CA06/0081.

Authors' disclosures of potential conflicts of interest and author contributions are found at the end of this article.

Corresponding author: Montserrat Ferrer, Institut Municipal d'Investigacions Mèdiques-Hospital del Mar, Health Services Research Group, Barcelona Biomedical Research Park (Office 144), Doctor Aiguader, 88, 08003 Barcelona, Spain; e-mail: mferrer@imim.es.

(C) 2010 by American Society of Clinical Oncology

$0732-183 X / 10 / 2831-4687 / \$ 20.00$

DOI: $10.1200 / J C O .2009 .25 .3245$

\title{
Quality-of-Life Impact of Primary Treatments for Localized Prostate Cancer in Patients Without Hormonal Treatment
}

Yolanda Pardo, Ferran Guedea, Ferrán Aguiló, Pablo Fernández, Víctor Macías, Alfonso Mariño, Asunción Hervás, Ismael Herruzo, María José Ortiz, Javier Ponce de León, Jordi Craven-Bratle, José Francisco Suárez, Ana Boladeras, Àngels Pont, Adriana Ayala, Gemma Sancho, Evelyn Martínez, Jordi Alonso, and Montserrat Ferrer

See accompanying editorial on page 4667

$\begin{array}{llllllll}\text { A } & \text { B } & \text { S } & \text { T } & \text { R } & \text { A } & \text { C } & \text { T }\end{array}$

\section{Purpose}

Earlier studies evaluating the effect on quality of life (QoL) of localized prostate cancer interventions included patients receiving adjuvant hormone therapy, which could have affected their outcomes. Our objective was to compare the QoL impact of the three most common primary treatments on patients who were not receiving adjuvant hormonal treatment.

\section{Patients and Methods}

This was a prospective study of 435 patients treated with radical prostatectomy, external-beam radiotherapy, or brachytherapy. OoL was assessed before and after treatment with the Short Form-36 and the Expanded Prostate Cancer Index Composite. Differences between groups were tested by analysis of variance. Distribution of outcome at 3 years was examined by stratifying according to baseline status. Generalized estimating equation models were constructed to assess the effect of treatment over time.

\section{Results}

Compared with the brachytherapy group, the prostatectomy group showed greater deterioration on urinary incontinence and sexual scores but better urinary irritative-obstructive results $(-18.22$, -13.19 , and +6.38 , respectively, at 3 years; $P<.001$ ). In patients with urinary irritative-obstructive symptoms at baseline, improvement was observed in $64 \%$ of those treated with nerve-sparing radical prostatectomy. Higher bowel worsening $(-2.87, P=.04)$ was observed in the external radiotherapy group, with $20 \%$ of patients reporting bowel symptoms.

\section{Conclusion}

Radical prostatectomy caused urinary incontinence and sexual dysfunction but improved preexisting urinary irritative-obstructive symptoms. External radiotherapy and brachytherapy caused urinary irritative-obstructive adverse effects and some sexual dysfunction. External radiotherapy also caused bowel adverse effects. Relevant differences between treatment groups persisted for up to 3 years of follow-up, although the difference in sexual adverse effects between brachytherapy and prostatectomy tended to decline over long-term follow-up. These results provide valuable information for clinical decision making.

\section{J Clin Oncol 28:4687-4696. (C) 2010 by American Society of Clinical Oncology}

\section{INTRODUCTION}

Prostate cancer is the most common cancer in men in the United States ${ }^{1}$ and the second most common in the European Union. ${ }^{2}$ Increased detection (incidence) associated with the widespread use of prostate-specific antigen testing has enabled diagnosis at earlier disease stages. ${ }^{1,3}$ A recent systematic review of localized prostate cancer treatment effectiveness ${ }^{4}$ concluded that all treatments caused urinary, bowel, or sexual dysfunction with different frequency, duration, and severity, but they were insufficiently characterized to facilitate clinical recommendations.

The occurrence of relevant treatment adverse effects combined with otherwise good results in terms of cancer control ${ }^{5-9}$ have led to a growing interest in evaluating the impact of treatment on quality of life (QoL). Previous longitudinal QoL studies ${ }^{10-15}$ included patients receiving hormone therapy, particularly as an adjunct to external or interstitial radiotherapy, which could have affected the results obtained for these primary treatments. Recently, Sanda et $\mathrm{al}^{14}$ showed that adjuvant 
androgen suppression exacerbated the adverse effects of external radiotherapy or brachytherapy on sexuality and vitality. Therefore, evidence is needed on the impact of the common primary treatments for localized prostate cancer on patients' QoL when used without hormone therapy.

Most longitudinal studies have only followed patients for up to $1^{16-18}$ or 2 years, ${ }^{13-15,19,20}$ whereas treatment adverse effects and QoL may change further with longer follow-up. ${ }^{10,11,21,22}$ Long-term studies could be particularly relevant in external or interstitial radiotherapy, as adverse effects might appear later as a result of chronic damage to adjacent tissues. Patients treated with external radiotherapy showed later worsening in incontinence ${ }^{21}$ and sexual dysfunction. ${ }^{1,21}$ However, the only long-term follow-up study that included brachytherapy showed recoveries ${ }^{21}$ in urinary irritative symptoms and bowel function.

The objective of this study was to compare the QoL impact of radical prostatectomy, three-dimensional external-beam radiotherapy, and prostate brachytherapy on patients with localized prostate cancer who were not receiving adjuvant hormonal treatment, from pretreatment to 3 years after the intervention.

\section{PATIENTS AND METHODS}

\section{Study Design and Participants}

This was a prospective study of a clinically localized prostate cancer cohort treated with radical prostatectomy, external-beam radiotherapy, or brachytherapy.

Participants included in the Spanish Multicentric Study of Clinically Localized Prostate Cancer were followed for 3 years after treatment. Details of the study are described elsewhere. ${ }^{15}$ Briefly, consecutive outpatients from 10 Spanish hospitals were enrolled from April 2003 to March 2005. Inclusion criteria were stages $\mathrm{T} 1$ or $\mathrm{T} 2^{23}$ and no previous transurethral prostate resection. For the purpose of this analysis, patients who received neoadjuvant or adjuvant hormonal therapy were excluded. Decisions on treatment options were made jointly by patients and physicians. The surgery group underwent radical retropubic prostatectomy, and the nerve-sparing technique was used at the surgeon's discretion. External-beam radiation was 3D conformal. Treatment was delivered with $1.8 \mathrm{~Gy}$ to $2.0 \mathrm{~Gy}$ daily fractions to a mean dose of $73.7 \mathrm{~Gy}$ (standard deviation $[\mathrm{SD}]=5.0$ ) to the prostate planning target volume. In the brachytherapy group, participants received ${ }^{125} \mathrm{I}$, and the prescription dose was 144 Gy to the reference isodose (100\%) according to the Task Group 43 (TG-T43). ${ }^{24}$ The median dose of D90 and V100\% was 158 Gy and $93 \%$, respectively.

Research protocols were approved by the ethics review boards of the participating hospitals, and written informed consent was required for each participant according to the 2000 revision of the Declaration of Helsinki.

\section{Measurement Instruments}

Participants' clinical characteristics evaluated at baseline included: $\mathrm{T}$ stage, prostate-specific antigen (PSA), Gleason histological grading scores, prostate volume, and reported chronic conditions. The definition of D'Amico et $\mathrm{al}^{5}$ was used to classify patients according to risk group. QoL instruments were administered centrally by telephone interviews before and 1, 3, 6, 12, 24, and 36 months after treatment.

Generic and prostate cancer-specific QoL questionnaires were included. The Short Form-36 Health Survey (SF-36) version $2^{25,26}$ was used. Scores for the two summary components (physical and mental component scores) were calculated using the recommended standardized procedure. ${ }^{25,26}$ The Expanded Prostate Cancer Index Composite (EPIC) ${ }^{27}$ includes 50 items grouped into two urinary subscales (incontinence and irritative-obstructive) and three summary scores (bowel, sexual, and hormonal), with scores ranging from 0 to 100. The International Prostate Symptom Score ${ }^{28,29}$ contains seven items measuring urinary obstruction, with scores ranging from 0 (no symptoms) to 35. With the exception of the International Prostate Symptom Score, higher scores indicate better QoL.

In addition to the EPIC continuous scores, we classified distressful levels of symptoms following the strategy proposed by Talcott et al "2,30 "No relevant problem" describes a patient with no distressful symptoms, "small to moderate problem" describes a patient reporting at least one distressful symptom, and "severe problem" describes a patient with at least one extremely distressful symptom. Within each EPIC domain, only the severity items were considered to construct this classification. These items and the level of distress associated with each response category are specified in the Appendix Table A1 (online only).

\section{Statistical Analysis}

Between-group differences on QoL mean scores at each assessment and changes in mean QoL scores (from baseline to 3 years after treatment) were tested using analysis of variance and Tukey's studentized range for post hoc comparisons.

To facilitate the interpretability of results, the percentages of patients with no relevant, small to moderate, and severe problems at 3 years after treatment were shown in bar charts. To examine the effect of pretreatment symptoms on the occurrence of treatment adverse effects, these bar charts were constructed after we stratified patients according to their symptoms severity at baseline. ${ }^{13}$

To assess the effect of treatment on QoL and adverse effects over time while accounting for correlation among repeated measures, we constructed separate generalized estimating equation (GEE) models for each EPIC scale and for the SF-36 physical component score (included as dependent variables). Age, risk group, and prostate volume at baseline were included in the models as adjusting factors. So as not to assume a linear association, we included time in the model as a categorical variable with four categories: pretreatment (reference), and months 12, 24, and 36. Regarding treatment, brachytherapy patients were used as the reference group in these models, and interactions between treatment and time were also included. The statistical analyses were carried out using SPSS 12.0 and SAS 9.1 software.

\section{RESULTS}

A total of 179 patients were excluded as a result of having received hormone treatment, resulting in a final sample of 435 patients (Table 1). There were statistically significant differences by treatment group at baseline for age, PSA, T stage, risk group, and prostate volume. Those receiving radical prostatectomy tended to be younger, whereas the lowest values for PSA, T stage, risk group, and prostate volume were seen in the prostate brachytherapy group. However, pretreatment QoL scores were similar among treatment groups.

Figure 1 shows QoL mean scores over the follow-up period by treatment group. Scores on the SF-36 mental component were quite stable, whereas a slight decrease was observed on the physical component. In the radical prostatectomy group, there was a notable decline in the urinary incontinence and sexual scores after treatment, with subsequent partial recovery. Sexual recovery was higher after nervesparing radical prostatectomy (NSRP) than after non-nerve-sparing radical prostatectomy (NNSRP). Compared with patients treated with surgery, the brachytherapy and external radiotherapy groups showed significantly lower (worse) urinary irritative-obstructive and bowel scores, respectively, during the last 2 years of follow-up. Mean changes in QoL scores from baseline to 3 years of follow-up are shown in Table 2. Sexual and urinary incontinence deterioration was greater in patients treated with radical prostatectomy (mean change, -23.9 and -25.1 , respectively) than in the other groups, though this group showed fewer changes in urinary irritative-obstructive symptoms. 
Table 1. Patient Characteristics and Quality-of-Life Scores at Pretreatment Evaluation and Response Rate at Each Follow-Up Assessment

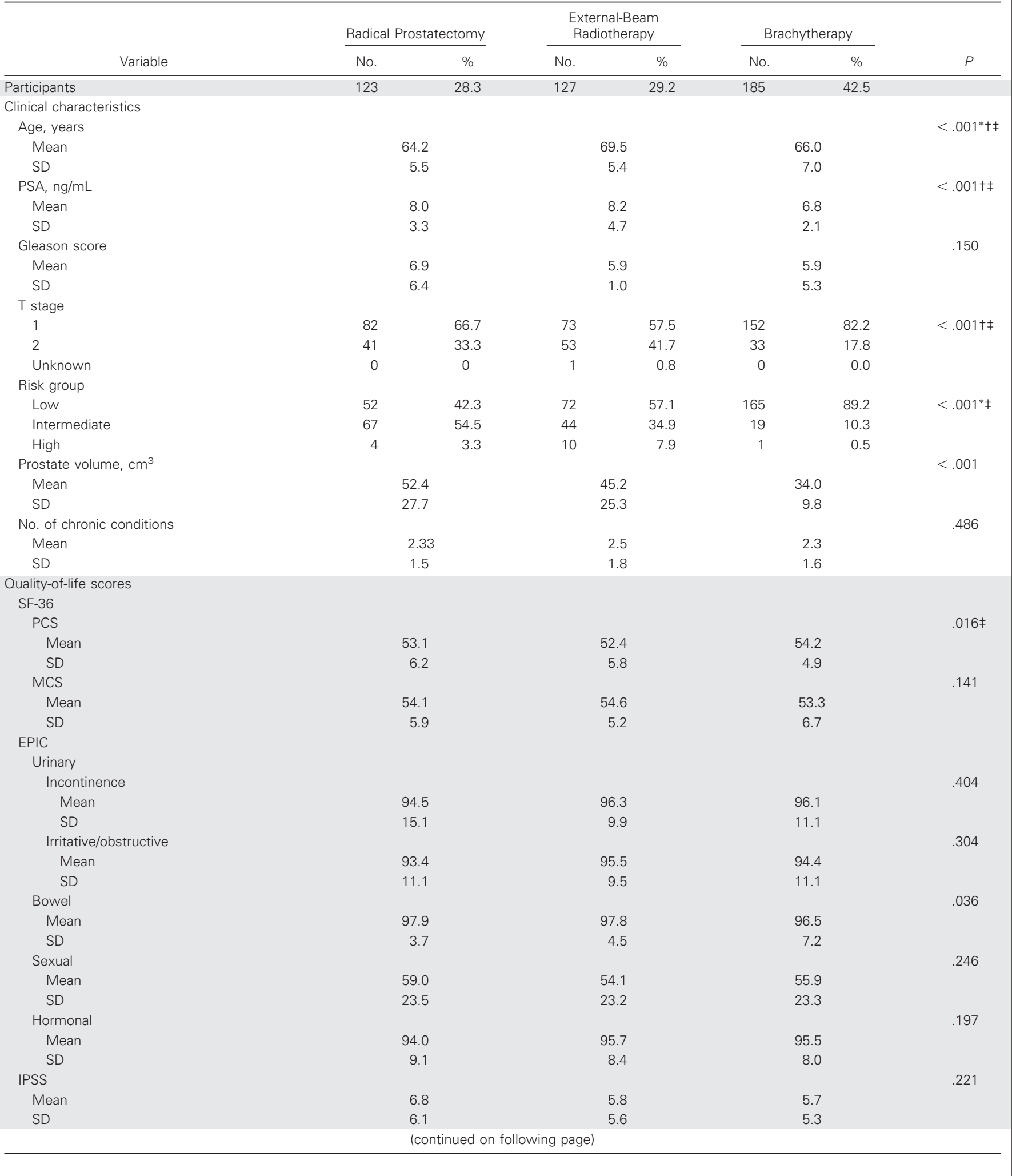


Table 1. Patient Characteristics and Quality-of-Life Scores at Pretreatment Evaluation and Response Rate at Each Follow-Up Assessment (continued)

\begin{tabular}{|c|c|c|c|c|c|c|c|}
\hline \multirow[b]{2}{*}{ Variable } & \multicolumn{2}{|c|}{ Radical Prostatectomy } & \multicolumn{2}{|c|}{$\begin{array}{c}\text { External-Beam } \\
\text { Radiotherapy }\end{array}$} & \multicolumn{2}{|c|}{ Brachytherapy } & \multirow[b]{2}{*}{$P$} \\
\hline & No. & $\%$ & No. & $\%$ & No. & $\%$ & \\
\hline \multicolumn{8}{|c|}{ Response rate (quality-of-life questionnaires) } \\
\hline Pretreatment & 123 & 100.0 & 127 & 100.0 & 185 & 100.0 & \\
\hline Month 1 & 66 & 53.7 & 43 & 33.9 & 100 & 54.1 & $.001^{*} \ddagger$ \\
\hline Month 3 & 114 & 92.7 & 117 & 92.1 & 172 & 93.0 & .961 \\
\hline Month 6 & 107 & 87.0 & 108 & 85.0 & 163 & 88.1 & .732 \\
\hline Month 12 & 111 & 90.2 & 112 & 88.2 & 169 & 91.4 & .654 \\
\hline Month 24 & 111 & 90.2 & 108 & 85.0 & 161 & 87.0 & .457 \\
\hline Month 36 & 109 & 88.6 & 100 & 78.7 & 155 & 83.8 & .107 \\
\hline \multicolumn{8}{|c|}{ 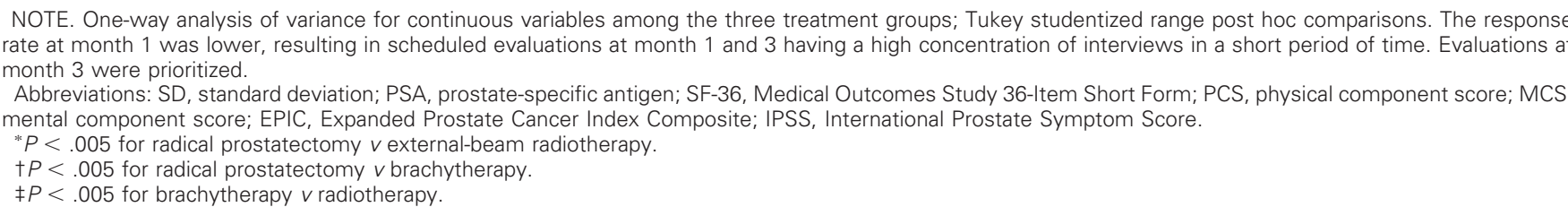 } \\
\hline
\end{tabular}

Bowel and hormonal deterioration were mainly observed in the radiotherapy group (mean change, -3.2 and -5.0 , respectively).

To expand the information represented by mean score changes, Figure 2 shows the distribution of patients with no relevant, small to moderate, and severe problems at 3 years according to baseline status, which indicates the proportion of patients who improved, preserved status, or worsened. Considering patients with no relevant sexual problem at baseline, approximately $40 \%$ of patients in the external and interstitial radiotherapy groups had preserved pretreatment sexual status compared with less than $10 \%$ in the surgery group; the proportion of patients with any severe problem after NNSRP (83\%) was higher than after NSRP (64\%). Among patients with any small to moderate sexual problem at baseline, deterioration was observed in $89 \%$ of patients after NNSRP and $67 \%$ after NSRP, compared with $50 \%$ for the brachytherapy group.

Because not enough patients $(n=9-18)$ had severe urinary or hormonal problems at baseline for a separate analysis, they were analyzed jointly with those patients who reported small to moderate problems. At 3 years, almost $75 \%$ of patients in the external and interstitial radiotherapy groups had preserved pretreatment urinary continence, compared with $45 \%$ of NSRP and $31 \%$ of NNSRP patients. Brachytherapy resulted in the highest proportion of patients who experienced urinary irritative-obstructive symptoms (35\%), whereas in patients with pretreatment small to severe urinary irritative-obstructive symptoms, improvement over baseline was observed in $64 \%$ of those treated with NSRP. Most of the patients did not present relevant bowel problems at baseline, and 23\% of patients treated with external radiotherapy reported bowel problems at 3 years. The proportion of patients with hormonal adverse effects was similar among treatment groups, approximately $20 \%$ in those with no relevant problem at baseline.

Table 3 shows the results from the GEE models constructed to assess treatment impact at different follow-up evaluations. In the urinary incontinence model, patients in the prostatectomy group showed significantly greater deterioration than those in the brachytherapy reference group throughout follow-up $(\beta=-20.10,-17.33$, and -18.22 at 1 st, 2 nd, and 3rd year, respectively; $P<.001)$. On irritative-obstructive symptoms, the difference from pretreatment (mean, 93.4; SD, 11.1) was significantly lower in the prostatectomy group at each year of follow-up $(\beta=+5.14,+5.56$, and +6.38 , respectively for 1st, 2 nd, and 3rd year), with positive coefficients indicating less deterioration from pretreatment than among patients in the brachytherapy group. In the sexual summary model, scores for patients in the brachytherapy group were lower (worse) than pretreatment, and deterioration increased ( $\beta=5.74,7.26$ and 10.03 , respectively, at the end of the 1st, $2 \mathrm{nd}$, and 3rd years). Deterioration from pretreatment was significantly larger in the prostatectomy group throughout follow-up $(P<.001)$, though differences with the brachytherapy group decreased somewhat over time $(\beta=21.30,19.74$ and 13.19 , respectively, at the 1st, $2 \mathrm{nd}$, and 3rd year).

Standard categorization of effect size (ES) was applied to understand the magnitude or clinical importance of these GEE $\beta$ coefficients. ES was calculated as adjusted mean differences $(\beta)$ divided by SD at baseline. The guidelines define an ES of 0.2 as small, 0.5 as moderate, and 0.8 as large. ${ }^{31,32}$ For example, the ES for significant differences between surgery and brachytherapy on the EPIC urinary incontinence, sexual, and urinary irritative-obstructive symptoms ( $\beta=18.2,13.2$, and +6.4 , respectively, at the 3rd year) was large for incontinence (ES, 1.2), and moderate for sexuality and urinary irritative-obstructive symptoms (ES, -0.6 and +0.6 ).

\section{DISCUSSION}

This comparative study of the three primary treatments for localized prostate cancer when used without hormone therapy shows a distinctive pattern of adverse effects for these treatments. Radical prostatectomy caused considerable urinary incontinence and sexual dysfunction, interstitial and external radiotherapy caused moderate urinary irritative-obstructive symptoms and sexual dysfunction, and external radiotherapy also produced moderate bowel-related adverse effects. Long-term modifications of adverse effects, such as an increase in urinary-related adverse effects after external radiotherapy or sexual adverse effects with brachytherapy, tended to reduce 


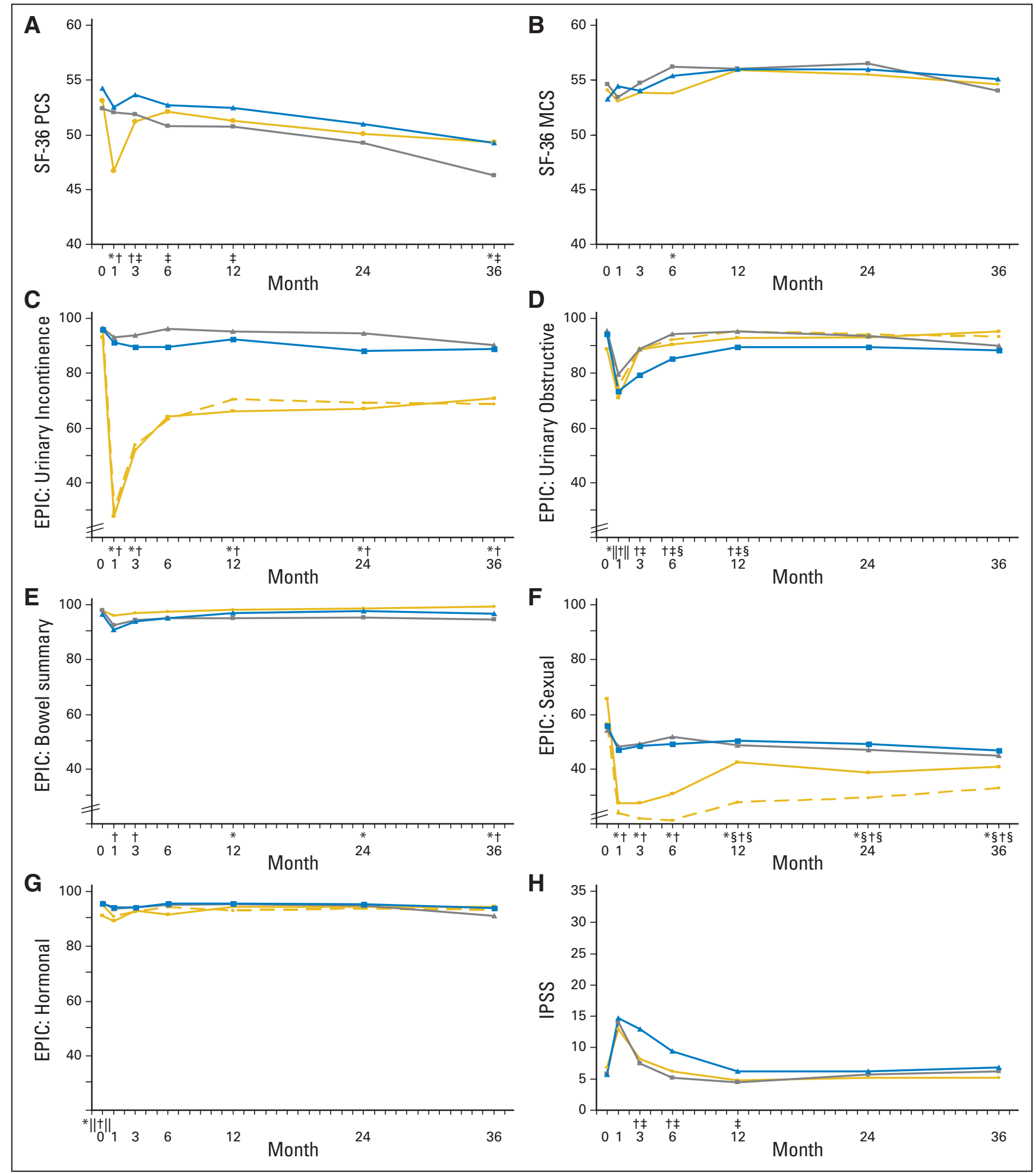

Fig 1. Mean quality-of-life (QoL) scores by treatment group-radical prostatectomy, external-beam radiotherapy, and brachytherapy-for Short Form-36 (SF-36) physical component score (PCS; A); SF-36 mental component score (MCS; B); Expanded Prostance Cancer Index Composite (EPIC) urinary incontinence (C), urinary obstructive (D), bowel (E), sexual (F), and hormonal (G) domains; and International Prostate Symptom Score (IPSS). The surgery group is shown separately, as a solid line for nerve-sparing radical prostatectomy (NSRP) and as a dashed line for non-nerve-sparing radical prostatectomy (NNSRP), for EPIC urinary, sexual, and hormonal domains. One-way analysis of variance of QoL scores among the three treatment groups for each follow-up assessment. Tukey studentized range post hoc comparisons: $\left.{ }^{*}\right) P<.05$ for radical prostatectomy versus external-beam radiotherapy: $(\dagger) P<.05$ for radical prostatectomy versus brachytherapy; ( $\left.¥\right) P<.05$ for brachytherapy versus radiotherapy; (§) $P<.05$ for non-nerve-sparing radical prostatectomy only; $(\|) P<.05$ for nerve-sparing radical prostatectomy only. 


\begin{tabular}{|c|c|c|c|c|c|c|c|c|c|}
\hline \multirow[b]{3}{*}{ Score } & \multicolumn{4}{|c|}{ Radical Prostatectomy } & \multirow{2}{*}{\multicolumn{2}{|c|}{$\begin{array}{c}\text { Conformal External } \\
\text { Radiotherapy } \\
\end{array}$}} & \multirow{2}{*}{\multicolumn{2}{|c|}{ Brachytherapy }} & \multirow[b]{3}{*}{$P$} \\
\hline & \multicolumn{2}{|c|}{ Non-Nerve Sparing } & \multicolumn{2}{|c|}{ Nerve Sparing } & & & & & \\
\hline & Mean & SD & Mean & SD & Mean & SD & Mean & SD & \\
\hline \multicolumn{10}{|l|}{ SF-36 PCS } \\
\hline PCS & -3.9 & 8.7 & -3.2 & 7.4 & -6.2 & 8.3 & -5.2 & 7.6 & .167 \\
\hline MCS & -0.2 & 9.7 & 2.2 & 7.8 & -0.7 & 9.1 & 1.3 & 8.8 & .215 \\
\hline \multicolumn{10}{|l|}{ EPIC } \\
\hline Urinary incontinence & -26.3 & 28.8 & -21.4 & 25.2 & -6.7 & 17.8 & -7.4 & 20.7 & $<.001^{*} \dagger$ \\
\hline Urinary irritative/obstructive & -1.8 & 14.4 & 7.0 & 13.1 & -5.6 & 15.9 & -5.9 & 21.0 & $.002 *$ キ†キ \\
\hline Bowel & 1.3 & 3.1 & 1.7 & 4.5 & -3.2 & 11.1 & 0.3 & 11.8 & $.006 * \S \|$ \\
\hline Sexual & -23.4 & 25.7 & -25.3 & 28.2 & -10.6 & 22.4 & -9.9 & 23.5 & $<.001^{*} \dagger$ \\
\hline Hormonal & -1.7 & 12.8 & 2.4 & 15.5 & -5.0 & 14.1 & -2.0 & 10.7 & $.034^{*} \ddagger$ \\
\hline IPSS & 0.9 & 7.0 & 2.7 & 4.7 & -0.4 & 6.3 & -1.1 & 7.0 & $.017 \dagger \ddagger$ \\
\hline \multicolumn{10}{|c|}{$\begin{array}{l}\text { One-way analysis of variance for the differences in quality-of-life scores from baseline to 3-year follow-up, by treatment group. Tukey studentized range post hoc } \\
\text { comparisons. } \\
\text { Abbreviations: SD, standard deviation; SF-36, Medical Outcomes Study 36-Item Short Form; PCS, physical component score; MCS, mental component score; EPIC, } \\
\text { Expanded Prostate Cancer Index Composite; IPSS, International Prostate Symptom Score. } \\
* P<.05 \text { for radical prostatectomy } v \text { radiotherapy. } \\
\dagger P<.05 \text { for radical prostatectomy } v \text { brachytherapy. } \\
\neq P<.05 \text { for nerve-sparing radical prostatectomy only. } \\
\S P<.05 \text { for non-nerve-sparing radical prostatectomy only. } \\
\| P<.05 \text { for brachytherapy } v \text { radiotherapy. }\end{array}$} \\
\hline
\end{tabular}

differences between treatments over time. However, these modifications were only slight and did not imply a real change in the characteristic pattern.

Sexual dysfunction was a common adverse effect of treatments, but it was also the most frequent concurrent symptom previous to treatment. In our sample, half of the patients presented severe sexual problems at baseline, almost $30 \%$ presented small to moderate problems, and only $22 \%$ did not present any relevant sexual problem. Differences in sexual adverse effects between patients with normal or poor pretreatment sexual functioning were reported previously. ${ }^{11,13,22}$ These studies showed that adverse effects were more severe in patients with better pretreatment sexual functioning, possibly because patients with sexual dysfunction may leave little margin for additional, treatment-related worsening. Consistent with this finding, out of the patients treated with surgery in our study, more than $90 \%$ of those without any relevant sexual problems at baseline presented sexual adverse effects, compared with $67 \%$ of patients with small to moderate sexual problems at baseline who received nerve-sparing procedures. Brachytherapy presented lower levels of sexual adverse effects than prostatectomy, with approximately half of patients worsening, independently of whether they experienced no relevant or small to moderate pretreatment sexual problems. These results provide useful evidence to inform patients regarding expected treatment adverse effects according to their levels of pretreatment sexual functioning.

Regarding urinary treatment-related effects, the prostatectomy group showed worse adverse effects for incontinence, but better results on urinary irritative-obstructive symptoms compared with brachytherapy at 3 years after treatment. The prostatectomy group presented approximately twice as many patients with urinary incontinence adverse effects (69\% for NNSRP and 54\% for NSRP), compared with $25 \%$ of those treated with external or interstitial radiotherapy without relevant pretreatment incontinence problems. Among patients with urinary obstructive-irritative symptoms at base- line, improvement was observed in $64 \%$ of patients treated with NSRP, $44 \%$ with brachytherapy, and $28 \%$ with external radiotherapy. Our results confirm previously reported improvement in urinary obstruction-irritation in patients treated with prostatectomy, and also for those treated with radiotherapy. ${ }^{14,22}$ It has been argued that external and interstitial radiotherapy may lead to small improvements because of a reduction in prostate size. This potential clinical benefit of radical prostatectomy may simplify clinical decision making for patients who have pre-existing irritative-obstructive urinary symptoms.

Only the group of patients treated with external radiotherapy presented a statistically significant moderate worsening of bowel symptoms (ES, -0.6). If we analyze these results at the individual level, ${ }^{33} 8 \%$ of patients presented severe bowel problems, and 15\% small to moderate ones, at 3 years. Finally, only a small deterioration in hormonal summary scores (ES, 0.2 ) was observed at 3 years. Although statistically significant differences in changes between treatment groups were observed in the bivariate analysis, they were not significant in the GEE model. Consistent with this finding, the distribution of patients with hormonal adverse effects was similar in the three treatment groups.

With regard to the long-term modification of adverse effects, it is noticeable that the patterns of urinary adverse effects in external radiotherapy and brachytherapy became more similar over time. During the first 2 years, positive coefficients in the incontinence and irritative-obstructive models indicated that patients treated with external radiotherapy had better results than those treated with brachytherapy. Nevertheless, the differences were no longer statistically significant at year $3(\beta=+5.2$ and .6 at 2 and 3 years, respectively, for incontinence). Worsening incontinence among patients treated with external radiotherapy was also reported by Miller et $\mathrm{al}^{21}$ from year 2 to year 6 of follow-up. The reduction of the differences in sexual dysfunction between the prostatectomy and brachytherapy groups was also noteworthy ( $\beta=-19.7$ and -13.2 at year 2 and year 3 , respectively). This reduction could be partly explained by the long-term sexual 


\section{A EPIC Sexual}

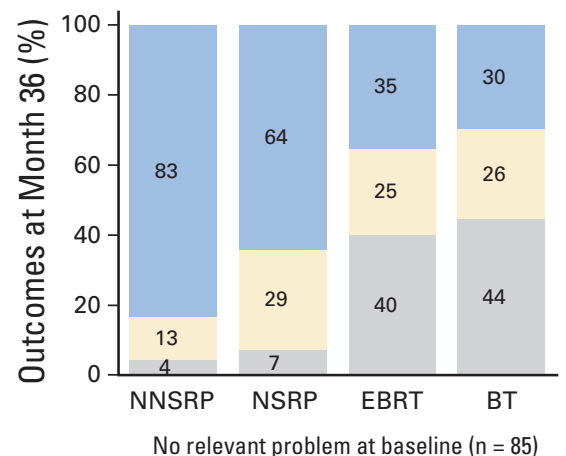

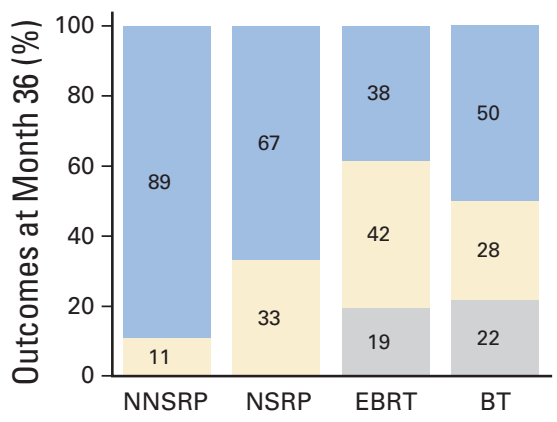

Small to moderate problem at baseline $(n=103)$

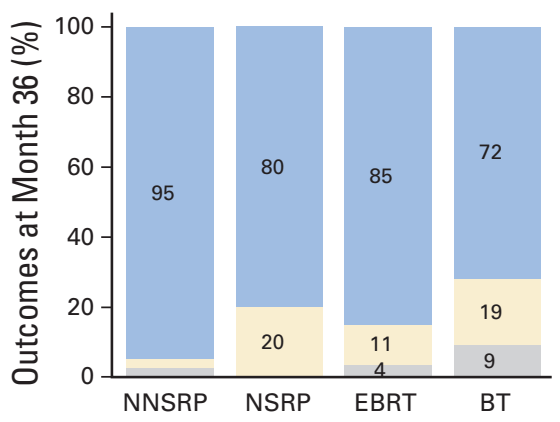

Severe problem at baseline $(n=176)$

\section{B EPIC Urinary Incontinence}
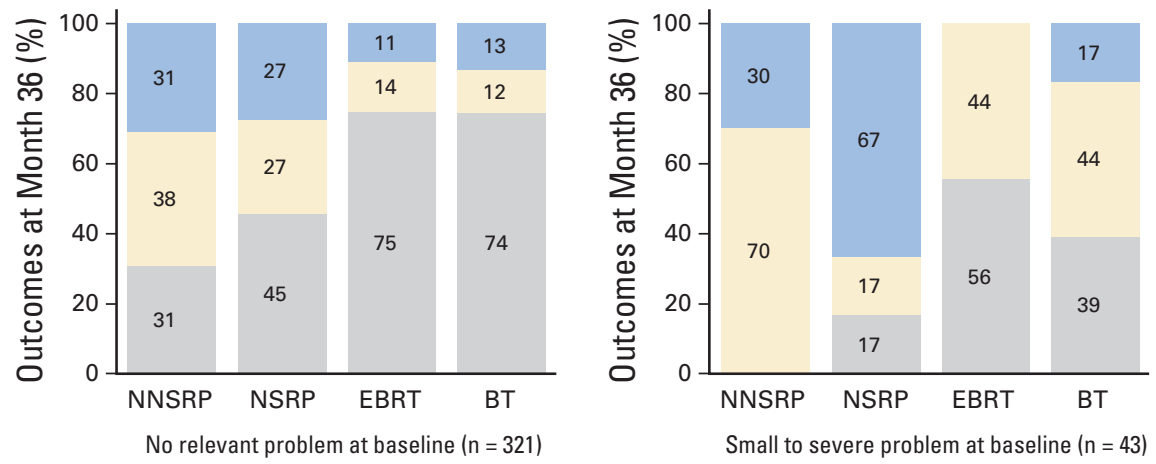

\section{EPIC Urinary Irritative / Obstructive}

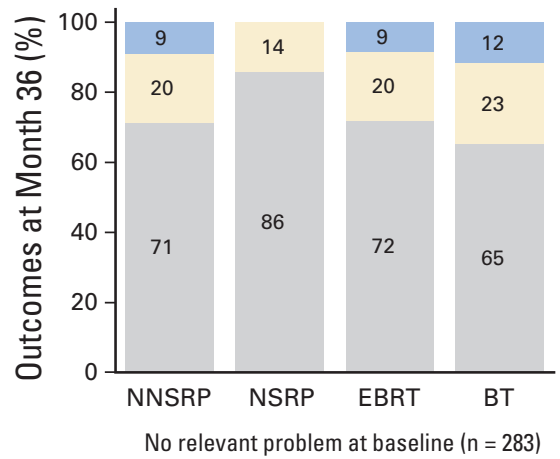

\section{EPIC Bowel}

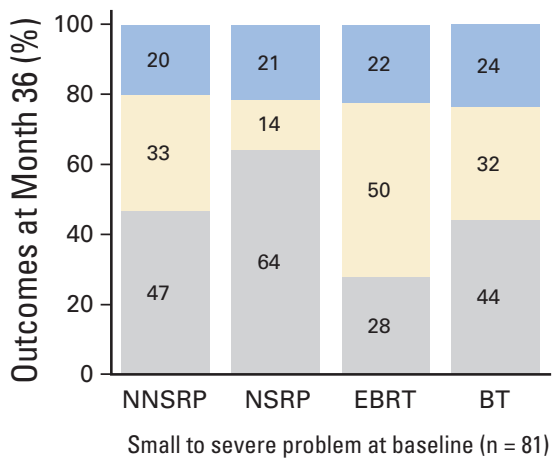

\section{E EPIC Hormonal}

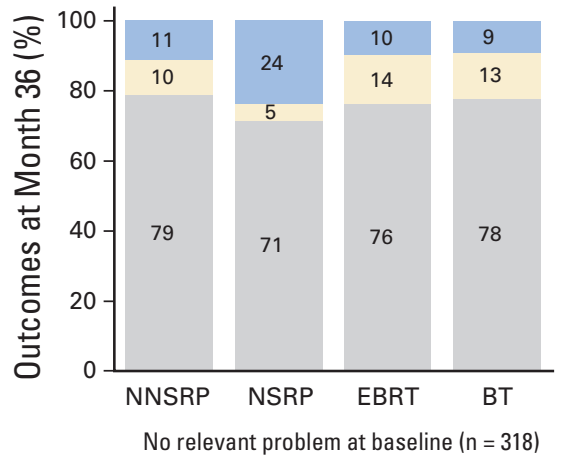

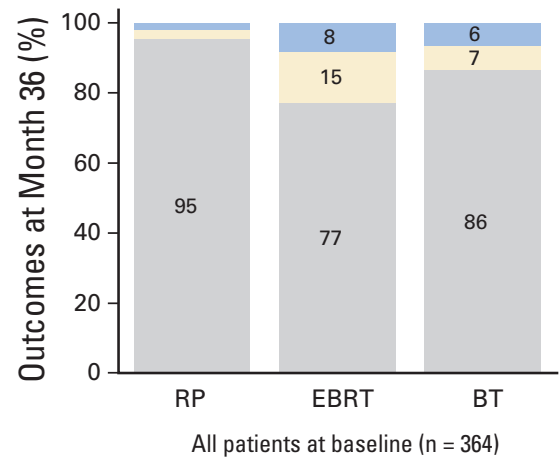

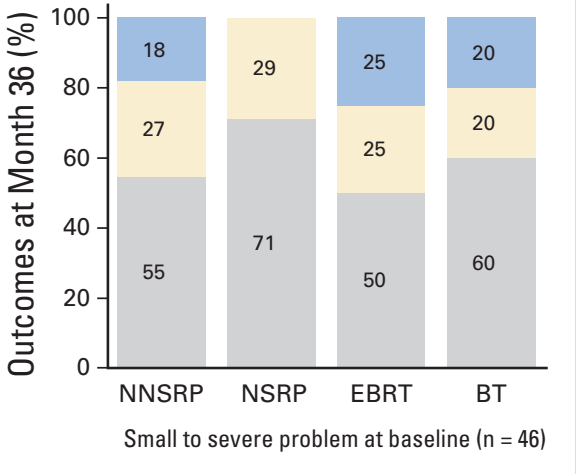

Fig 2. Distribution of patients with no relevant problems (light gray), small to moderate problems (light tan), or severe problems (light blue) at 3 years after treatment, stratified by baseline symptoms on Expanded Prostate Cancer Index Composite (A) sexual, (B) urinary incontinence, (C) urinary irritative-obstructive, (D) bowel, and (E) hormonal domains. EPIC, Expanded Prostate Cancer Index Composite; NNSRP, non-nerve-sparing radical prostatectomy; NSRP, nerve-sparing radical prostatectomy; EBRT, external-beam radiation therapy; BT, brachytherapy; RP, radical prostatectomy. Sample size was not large enough to perform bowel outcome analysis of the groups with small to moderate problems $(n=14)$ and severe problems $(n=5)$ at baseline separately. 
Table 3. Generalized Estimating Equation Models of the Association Between Treatment Groups and Clinical Variables, With SF-36 Physical Component Score and EPIC Scores at Each Year of Follow-Up

\begin{tabular}{|c|c|c|c|c|c|c|c|c|c|}
\hline \multirow[b]{2}{*}{ Variable } & \multicolumn{3}{|c|}{$\begin{array}{l}\text { SF-36 Physical Component } \\
\text { Score } \\
\end{array}$} & \multicolumn{3}{|c|}{ EPIC Urinary Incontinence } & \multicolumn{3}{|c|}{$\begin{array}{l}\text { EPIC Urinary Irritative- } \\
\text { Obstructive }\end{array}$} \\
\hline & $\beta$ & SE & $P$ & $\beta$ & SE & $P$ & $\beta$ & SE & $P$ \\
\hline Intercept & 63.29 & 2.53 & $<.0001$ & 89.1 & 9.29 & $<.0001$ & 98.12 & 5.99 & $<.0001$ \\
\hline Age & -0.14 & 0.04 & $<.0001$ & 0.08 & 0.14 & .552 & -0.06 & 0.09 & .507 \\
\hline Prostate volume & 0 & 0.01 & .979 & 0.03 & 0.04 & .363 & 0 & 0.02 & .993 \\
\hline \multicolumn{10}{|l|}{ Risk group } \\
\hline Low & \multicolumn{2}{|c|}{ ref. } & ref. & \multicolumn{2}{|c|}{ ref. } & ref. & \multicolumn{2}{|c|}{ ref. } & ref. \\
\hline Intermediate-high & 0.26 & 0.63 & .682 & 2.73 & 1.77 & .122 & 1.17 & 1.11 & .29 \\
\hline \multicolumn{10}{|c|}{ Treatment group (differences at baseline) } \\
\hline Brachytherapy & \multicolumn{2}{|c|}{ ref. } & ref. & \multicolumn{2}{|c|}{ ref. } & ref. & \multicolumn{2}{|c|}{ ref. } & ref. \\
\hline Radiotherapy & -1.67 & 0.71 & 0.018 & -0.64 & 1.45 & .66 & 0.83 & 1.41 & .554 \\
\hline Prostatectomy & -1.75 & 0.78 & 0.026 & -2.8 & 1.92 & .146 & -1.27 & 1.55 & .412 \\
\hline \multicolumn{10}{|c|}{$\begin{array}{l}\text { Interaction brachytherapy } \times \text { time } \\
\text { (change from baseline) }\end{array}$} \\
\hline Baseline & \multicolumn{2}{|c|}{ ref. } & ref. & \multicolumn{2}{|c|}{ ref. } & ref. & \multicolumn{2}{|c|}{ ref. } & ref. \\
\hline 12 months & -1.73 & 0.4 & $<.0001$ & -3.75 & 1.34 & .005 & -4.62 & 1.29 & 0 \\
\hline 24 months & -3.26 & 0.56 & $<.0001$ & -7.58 & 1.58 & $<.0001$ & -4.8 & 1.4 & .001 \\
\hline 36 months & -4.93 & 0.57 & $<.0001$ & -6.85 & 1.61 & $<.0001$ & -5.73 & 1.64 & .001 \\
\hline \multicolumn{10}{|c|}{$\begin{array}{l}\text { Interaction radiotherapy } \times \text { time } \\
\quad \text { (difference from brachytherapy) }\end{array}$} \\
\hline Baseline & \multicolumn{2}{|c|}{ ref. } & ref. & \multicolumn{2}{|c|}{ ref. } & ref. & \multicolumn{2}{|c|}{ ref. } & ref. \\
\hline 12 months & 0.17 & 0.7 & .811 & 2.15 & 1.85 & .245 & 4.21 & 1.64 & .01 \\
\hline 24 months & 0.44 & 0.91 & .631 & 5.16 & 2.07 & .013 & 2.77 & 1.87 & .139 \\
\hline 36 months & -0.75 & 1.03 & .467 & -0.63 & 2.48 & .798 & 0.06 & 2.42 & .981 \\
\hline
\end{tabular}

Interaction prostatectomy $\times$ time (difference from brachytherapy)

Baseline

12 months

ref. .467

5.16
-0.63

2.48

.013

24 months

$0.01 \quad 0.78$

ref.

$0.64 \quad 1.05$

.985
.889
.544

\begin{tabular}{lr}
\multicolumn{2}{r}{ ref. } \\
-20.1 & 3.31 \\
-17.33 & 3.32 \\
-18.22 & 3.08
\end{tabular}

ref.

$<.0001$

$<.0001$

$<.0001$

$5.14^{\text {ref }}$

5.562

6.38

1.87
2

ref.

36 months

EPIC Bowel Summary EPIC Sexual Summary

EPIC Hormonal Summary

\begin{tabular}{l} 
Variable \\
\hline Intercept \\
Age \\
Prostate volume \\
Risk group
\end{tabular}

$$
\text { Low }
$$

Intermed.-high

$\beta \quad$ SE

Treatment group (differences at baseline) Brachytherapy

Radiotherapy

Prostatectomy

$93.74 \quad 2.74$

$0.04 \quad 0.04$

0.01

ref.

$\begin{array}{rrr}<.0001 & 106.53 & 9.98 \\ .283 & -0.77 & 0.15 \\ .672 & 0.01 & 0.04\end{array}$

EPIC Sexual Summary
$\beta$ SE

$P$

-

EPIC Hormonal Summary

nteraction brachytherapy $\times$ time

(change from baseline)

Baseline

12 months

$1.07 \quad 0.51$

ref.

24 months

36 months

ref.

ref. ref

$\begin{array}{lll}0.88 & 0.76 & .245 \\ 1.12 & 0.72 & .12\end{array}$

.245
.12

035

0.9

ref.

.15

$<.0001$

$<.0001$

85.39

85.39
0.16

$-0.01$

SE

\begin{tabular}{cc} 
SE & $P$ \\
\hline 3.98 & $<.0001$ \\
0.06 & .005
\end{tabular}

Interaction radiotherapy $\times$ time (difference from brachytherapy)

\section{Baseline}

12 months

24 months

1.12

45

$\begin{array}{ll} & \text { ref. } \\ 0.51 & 0.68 \\ 1.19 & 0.61 \\ 0.2 & 0.91\end{array}$

ref.
.454
.051
827

ref.
.454

.051

.827

\begin{tabular}{ll}
\multicolumn{2}{r}{ ref. } \\
1.51 & 2.96 \\
0.4 & 3.15
\end{tabular}

21)

ref.

$0.3^{\text {ref. }}$

0.02

.005

0.2

0.91

ref.

$-2.99 \quad 1.13$

ref.

.008

.001

.04

$-2.87$

1.2
1.4

ref.
-5.74
-7.26
-10.03

ref.

1.72
1.84
1.85

ref.

Interaction prostatectomy $\times$ time

(difference from brachytherapy)

Baseline

12 months

\begin{tabular}{ll} 
& \multicolumn{2}{c}{ ref. } \\
-0.19 & 0.96 \\
-0.46 & 0.73 \\
1.15 & 0.98
\end{tabular}

ref.

.843

\begin{tabular}{ll}
\multicolumn{2}{c}{ ref. } \\
-1.06 & 2.57 \\
-1.67 & 2.88
\end{tabular}

$\begin{array}{ll}-1.23 & 3.07\end{array}$

$\begin{array}{ll}.685 & \\ \text { ref. } & \\ .61 & -0.63 \\ .9 & -1.5\end{array}$

ef. 0

0.83

ref.

24 months

$\begin{array}{ll}1.15 & 0.98\end{array}$

.53

.239

\begin{tabular}{ll}
\multicolumn{2}{r}{ ref. } \\
-21.3 & 3.16 \\
-19.74 & 3.34 \\
-13.19 & 3.28
\end{tabular}

ref.

$<.0001$

$<.0001$

$<.0001$

$\begin{array}{ll}\text { ref. } & -0.68 \\ .68 & \text { ref. }\end{array}$

$.563-0.14$

1.15

1.15

1.15
1.13

NOTE Time was included in the model as a categorical variable with four categories so as not to assume a linear association: baseline (reference), month 12 , month 24 , and month 36. Interaction variables between each treatment group and time were included: for interaction with brachytherapy, the coefficients refer to the

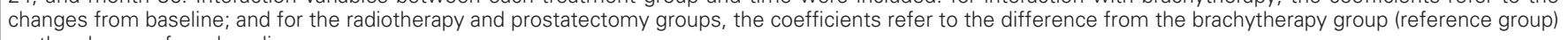

on the changes from baseline.

Abbreviations: SF-36, Short Form-36; EPIC, Expanded Prostate Cancer Index Composite; ref., reference. 
deterioration observed in brachytherapy patients $(\beta=-7.3$ and -10.0 at year 2 and year 3 ). Chen et $\mathrm{al}^{22}$ also reported a slight tendency to deteriorate on this parameter in the brachytherapy group, compared with a tendency toward recovery in the prostatectomy group. A longer follow-up would be needed to confirm whether the worsening observed in our sample from the 2nd to the 3rd year of follow-up indicates a true trend toward later occurrence of sexual dysfunction with brachytherapy treatment.

Some limitations of this study should be taken into account. First, this is an observational study, and participants were not randomly assigned to treatment groups. However, randomized clinical trials have presented considerable difficulties in these patients. ${ }^{34}$ Baseline differences in clinical characteristics between treatment groups in our sample could be attributed to the observational design of the study. To account for the possible effect of clinical differences, we adjusted GEE models by the relevant clinical characteristics. Age, risk group, or prostate volume showed little impact on treatment outcomes. Second, nerve-sparing techniques were not widely applied in our study $(28 \%$ of patients treated with prostatectomy), and our findings from the GEE model for the prostatectomy group as a whole could overestimate their adverse sexual effects. Nevertheless, large sexual adverse effects for prostatectomy treatment were also shown by other studies that included high proportions of patients who received nerve-sparing procedures. ${ }^{13,14}$ Third, improvements observed in urinary incontinence, hormonal function, and sexual function, regardless of which treatment was applied, were generally experienced by a low number of patients, and may be partially explained by the regression to the mean phenomenon. Unexpected improvements have also been reported in previous studies. ${ }^{11,35}$

This study provides (to our knowledge) novel long-term results on QoL and adverse effects for the three common primary treatments in patients with localized prostate cancer who were not receiving adjunct hormonal treatment. Furthermore, the interpretation strategy addressed at the individual and aggregate levels followed here provides complementary useful information. Mean scores of treatment groups indicate results on average and facilitate comparison with other studies because it is the most usual approach. However, it could be a challenge to communicate the evidence to patients in a meaningful way. In fact, clinical interpretability of QoL scores has been identified as one of the barriers to the use of this type of measure. ${ }^{36}$ The inter- pretation approach at an individual level shows the percentages of patients with adverse effects, and could facilitate clinical interpretation and the transmission of information to patients.

In conclusion, our findings suggest that adverse effects of external and interstitial radiotherapy could increase beyond 2 years of followup. Nevertheless, urinary irritative-obstructive symptoms, sexual dysfunction, and bowel-related adverse effects associated with interstitial or external radiotherapy were still moderate at 3 years of follow-up, whereas radical prostatectomy was associated with substantial urinary incontinence and sexual dysfunction over the same period. These results could provide relevant information to characterize adverse effects of primary treatments and facilitate shared clinical decision making between patients and professionals.

\section{AUTHORS' DISCLOSURES OF POTENTIAL CONFLICTS OF INTEREST}

The author(s) indicated no potential conflicts of interest.

\section{AUTHOR CONTRIBUTIONS}

Conception and design: Ferran Guedea, Ferrán Aguiló,

Montserrat Ferrer

Provision of study materials or patients: Ferran Guedea, Ferrán Aguiló, Pablo Fernández, Víctor Macías, Alfonso Mariño, Asunción Hervás, Ismael Herruzo, María José Ortiz, Javier Ponce de León, Jordi Craven-Bratle, José Francisco Suárez, Ana Boladeras, Adriana Ayala, Gemma Sancho, Evelyn Martínez, Montserrat Ferrer

Collection and assembly of data: Ferran Guedea, Ferrán Aguiló, Pablo Fernández, Víctor Macías, Alfonso Mariño, Asunción Hervás, Ismael Herruzo, María José Ortiz, Javier Ponce de León, Jordi Craven-Bratle, José Francisco Suárez, Ana Boladeras, Àngels Pont, Adriana Ayala, Gemma Sancho, Evelyn Martínez, Montserrat Ferrer

Data analysis and interpretation: Yolanda Pardo, Ferran Guedea, Àngels Pont, Jordi Alonso, Montserrat Ferrer

Manuscript writing: Yolanda Pardo, Ferran Guedea, Jordi Alonso, Montserrat Ferrer

Final approval of manuscript: Yolanda Pardo, Ferran Guedea, Ferrán Aguiló, Pablo Fernández, Víctor Macías, Alfonso Mariño, Asunción Hervás, Ismael Herruzo, María José Ortiz, Javier Ponce de León, Jordi Craven-Bratle, José Francisco Suárez, Ana Boladeras, Àngels Pont, Adriana Ayala, Gemma Sancho, Evelyn Martínez, Jordi Alonso, Montserrat Ferrer

\section{REFERENCES}

1. Jemal $A$, Siegel $R$, Ward $E$, et al: Cancer statistics, 2008. CA Cancer J Clin 58:71-96, 2008

2. Bray F, Sankila R, Ferlay J, et al: Estimates of cancer incidence and mortality in Europe in 1995. Eur J Cancer 38:99-166, 2002

3. Sant $M$, Aareleid $T$, Berrino $F$, et al: EUROCARE-3: Survival of cancer patients diagnosed 199094-results and commentary. Ann Oncol 14:v61-v118, 2003 (suppl 5)

4. Wilt TJ, MacDonald R, Rutks I, et al: Systematic review: Comparative effectiveness and harms of treatments for clinically localized prostate cancer. Ann Intern Med 148:435-448, 2008

5. D'Amico AV, Whittington R, Malkowicz SB, et al: Biochemical outcome after radical prostatectomy, external beam radiation therapy, or interstitial radiation therapy for clinically localized prostate cancer. JAMA 280:969-974, 1998
6. Polascik TJ, Pound CR, DeWeese TL, et al: Comparison of radical prostatectomy and iodine 125 interstitial radiotherapy for the treatment of clinically localized prostate cancer: A 7-year biochemical (PSA) progression analysis. Urology 51: 884-889, 1998

7. Ramos CG, Carvalhal GF, Smith DS, et al: Retrospective comparison of radical retropubic prostatectomy and 125iodine brachytherapy for localized prostate cancer. J Urol 161:1212-1215, 1999

8. Stokes SH: Comparison of biochemical diseasefree survival of patients with localized carcinoma of the prostate undergoing radical prostatectomy, transperineal ultrasound-guided radioactive seed implantation, or definitive external beam irradiation. Int J Radiat Oncol Biol Phys 47:129-136, 2000

9. Oliva G: Braquiterapia en Cáncer de Próstata :Informe Técnico de de la Agència d'Avaluació de Tecnologia i Recerca Mèdiques (Noviembre 2000). Madrid, Spain, Ministerio de Sanidad y Consumo, 2001
10. Korfage IJ, Essink-Bot ML, Borsboom GJ, et al: Five-year follow-up of health-related quality of life after primary treatment of localized prostate cancer. Int J Cancer 116:291-296, 2005

11. Potosky AL, Davis WW, Hoffman RM, et al: Five-year outcomes after prostatectomy or radiotherapy for prostate cancer: The prostate cancer outcomes study. J Natl Cancer Inst 96:1358-1367, 2004

12. Hoffman RM, Gilliland FD, Penson DF, et al: Cross-sectional and longitudinal comparisons of health-related quality of life between patients with prostate carcinoma and matched controls. Cancer 101:2011-2019, 2004

13. Litwin MS, Gore JL, Kwan L, et al: Quality of life after surgery, external beam irradiation, or brachytherapy for early-stage prostate cancer. Cancer 109:2239-2247, 2007

14. Sanda MG, Dunn RL, Michalski J, et al: Quality of life and satisfaction with outcome among prostatecancer survivors. N Engl J Med 358:1250-1261, 2008 
15. Ferrer M, Suárez JF, Guedea $F$, et al: Healthrelated quality of life 2 years after treatment with radical prostatectomy, prostate brachytherapy, or external beam radiotherapy in patients with clinically localized prostate cancer. Int J Radiat Oncol Biol Phys 72:421-432, 2008

16. Krupski T, Petroni GR, Bissonette EA, et al: Quality-of-life comparison of radical prostatectomy and interstitial brachytherapy in the treatment of clinically localized prostate cancer. Urology 55:736742, 2000

17. Borchers $H$, Kirschner-Hermanns $R$, Brehmer $B$, et al: Permanent 125l-seed brachytherapy or radical prostatectomy: A prospective comparison considering oncological and quality of life results. BJU Int 94:805-811, 2004

18. Schapira MM, Lawrence WF, Katz DA, et al: Effect of treatment on quality of life among men with clinically localized prostate cancer. Med Care 39:243-253, 2001

19. Talcott JA, Manola J, Clark JA, et al: Time course and predictors of symptoms after primary prostate cancer therapy. J Clin Oncol 21:3979-3986 2003

20. Downs TM, Sadetsky N, Pasta DJ, et al: Health related quality of life patterns in patients treated with interstitial prostate brachytherapy for localized prostate cancer-Data from CaPSURE. J Urol 170:1822-1827, 2003

21. Miller DC, Sanda MG, Dunn RL, et al: Longterm outcomes among localized prostate cance survivors: Health-related quality-of-life changes after radical prostatectomy, external radiation, and brachytherapy. J Clin Oncol 23:2772-2780, 2005

22. Chen RC, Clark JA, Talcott JA: Individualizing quality-of-life outcomes reporting: How localized prostate cancer treatments affect patients with different levels of baseline urinary, bowel, and sexua function. J Clin Oncol 27:3916-3922, 2009

23. American Joint Committee on Cancer: Cancer Staging Manual. New York, NY, Springer, 2002

24. Bice WS Jr, Prestidge BR, Prete JJ, et al: Clinical impact of implementing the recommendations of AAPM Task Group 43 on permanent prostate brachytherapy using 125l. American Association of Physicists in Medicine. Int J Radiat Oncol Biol Phys 40:1237-1241, 1998

25. Ware JE Jr, Kosinski M, Turner-Bowker DM, et al: How to Score Version 2 of the SF-12 Health Survey. Lincoln, RI, QualityMetric, 2002

26. Vilagut $G$, Ferrer $M$, Rajmil $L$, et al: El cuestionario de salud SF-36 español: Una década de experiencia y nuevos desarrollos. Gac Sanit 19:135150,2005

27. Wei JT, Dunn RL, Litwin MS, et al: Development and validation of the Expanded Prostate Cancer Index Composite (EPIC) for comprehensive assessment of health-related quality of life in men with prostate cancer. Urology 56:899-905, 2000

28. Badia X, Garcia-Losa M, Dal Re R, et al: Validation of a harmonized Spanish version of the IPSS: Evidence of equivalence with the original American scale. Urology 52:614-620, 1998
29. Badia X, Garcia-Losa M, Dal Re R: Tenlanguage translation and harmonization of the Inter national Prostate Symptom Score: Developing a methodology for multinational clinical trials. Eur Urol 31:129-140, 1997

30. Talcott JA, Clark JA, Manola J, et al: Bringing prostate cancer quality of life research back to the bedside: Translating numbers into a format that patients can understand. J Urol 176:1558-1563, 2006

31. Cohen J: Statistical Power Analysis for the Behavioral Sciences. Hillsdale, NJ, Lawrence Erlbaum Associates, 1988

32. Kazis LE, Anderson JJ, Meenan RF: Effect sizes for interpreting changes in health status. Med Care 27:S178-S189, 1989

33. Guyatt G, Schunemann H: How can quality of life researchers make their work more useful to health workers and their patients? Qual Life Res 2007

34. Wilt TJ: Can randomized treatment trials in early stage prostate cancer be completed? Clin Oncol 10:141-143, 1998

35. Litwin MS, Sadetsky N, Pasta DJ, et al: Bowe function and bother after treatment for early stage prostate cancer: A longitudinal quality of life analysis from CaPSURE. J Urol 172:515-519, 2004

36. Scientific Advisory Committee of the Medical Outcomes Trust: Assessing health status and quality-of-life instruments: Attributes and review criteria. Qual Life Res 11:193-205, 2002 


\section{CORRECTIONS}

\section{Author Corrections}

The November 20, 2010, special article by Pappo et al, entitled "Infrequent Tumor Initiative of the Children's Oncology Group: Initial Lessons Learned and Their Impact on Future Plans" (J Clin Oncol 28:5011-5016, 2010), contained an error.

In the Introduction section, the second sentence was given as: "Indeed, the Rare Disease Act of $2002^{1}$ defines a rare disease as one that affects fewer than 200,000 persons per year in the United States."
Whereas it should have read:

"Indeed, the Rare Disease Act of $2002^{1}$ defines a rare disease as one that affects fewer than 200,000 persons in the United States."

The authors apologize to the readers for the mistake.

DOI: $10.1200 /$ JCO.2011.34.7351
The December 20, 2010, article by Kunitake et al, entitled "Routine Preventive Care and Cancer Surveillance in Long-Term Survivors of Colorectal Cancer: Results From National Surgical Adjuvant Breast and Bowel Project Protocol LTS-01" (J Clin Oncol 28:5274-5279, 2010), contained an error.
The first initial of the seventh author's name was inadvertently omitted. The author's name was given as Lawrence Wickerham and should have been D. Lawrence Wickerham.

The authors apologize to the readers for the mistake.

DOI: $10.1200 /$ JCO.2011.34.7369

\section{Journal Corrections}

The November 1, 2010, article by Pardo et al, entitled "Quality-of-Life Impact of Primary Treatments for Localized Prostate Cancer in Patients Without Hormonal Treatment" (J Clin Oncol 28:4687-4696, 2010), contained an error.

In the legend of Figure 1, the colors of the lines corresponding to the treatments were inadvertently omitted. The corrected legend is reprinted below in its entirety.

Mean quality-of-life (QoL) scores by treatment groupradical prostatectomy (gold), external-beam radiotherapy (gray), and brachytherapy (blue)_for (A) Short Form-36 (SF36) physical component score (PCS); (B) SF-36 mental component score (MCS); (C) Expanded Prostate Cancer Index Composite (EPIC) urinary incontinence, (D) urinary obstructive, (E) bowel, (F) sexual, and (G) hormonal domains; and International Prostate Symptom Score (IPSS). The surgery group is shown separately, as a solid line for nerve-sparing radical prostatectomy (NSRP) and as a dashed line for nonnerve-sparing radical prostatectomy (NNSRP), for EPIC urinary, sexual, and hormonal domains. One-way analysis of variance of QoL scores among the three treatment groups for each follow-up assessment. Tukey studentized range post hoc comparisons: $\left.{ }^{*}\right) P<.05$ for radical prostatectomy versus external-beam radiotherapy; $(\dagger) P<.05$ for radical prostatectomy versus brachytherapy; ( ) $P<.05$ for brachytherapy versus radiotherapy; (\$) $P<.05$ for NNSRP only; (\|) $P<.05$ for NSRP only.

Journal of Clinical Oncology apologizes to the authors and readers for the mistake.

DOI: $10.1200 /$ JCO.2011.34.7377
The May 10, 2010, article by Ziepert et al, entitled "Standard International Prognostic Index Remains a Valid Predictor of Outcome for Patients With Aggressive CD20 ${ }^{+}$B-Cell Lymphoma in the Rituximab Era" (J Clin Oncol 28:2373-2380, 2010), contained an error.

The institutional affiliation for Evelyn Kuhnt should have been given as the Clinical Trial Centre, Universität Leipzig, Leipzig, Germany.

Journal of Clinical Oncology apologizes to the authors and readers for the mistake.

DOI: $10.1200 /$ JCO.2011.34.7393 